\title{
Technical principles of computed tomography in patients with congenital heart disease
}

\author{
Bjoern Stinn • Paul Stolzmann • Juergen Fornaro • \\ Dennis Hibbeln • Hatem Alkadhi • Simon Wildermuth • \\ Sebastian Leschka
}

Received: 28 August 2010 / Revised: 8 December 2010 / Accepted: 10 March 2011 / Published online: 3 April 2011

(C) European Society of Radiology 2011

\begin{abstract}
Cardiac magnetic resonance imaging and echocardiography are often the primary imaging techniques for many patients with congenital heart disease (CHD). However, with modern generations of CT systems and recent advances in temporal and spatial resolution, cardiac $\mathrm{CT}$ has been gaining an increasing reputation in the field of cardiac imaging and in the evaluation of patients with congenital heart disease. The CT imaging protocol depends on the suspected cardiac defect, the type of previous surgical repair, and the patient's age and level of cooperation. Various strategies are available for reducing radiation exposure, which is of utmost importance particularly in paediatric patients. A sequential segmental analysis is a commonly used approach to analysing congenital heart defects. Familiarity of the performing radiologist with dedicated CT protocols, the complex anatomy, morphology and terminology of CHD, as well as with the surgical procedures used to correct congenital abnormalities is a prerequisite for correct diagnosis.
\end{abstract}

Keywords Computed tomography · Cardiac CT · Coronary angiography $\cdot$ Congenital heart disease $\cdot$ Sequential segmental analysis

\footnotetext{
B. Stinn · J. Fornaro - D. Hibbeln · S. Wildermuth •

S. Leschka $(\triangle)$

Institute of Radiology, General Hospital Saint Gall,

Rorschacherstrasse 95,

9007 St Gallen, Switzerland

e-mail: sebastian.leschka@kssg.ch

P. Stolzmann $\cdot$ H. Alkadhi

Cardiac MR PET CT Program, Massachusetts General Hospital,

Harvard Medical School,

Boston, MA, USA
}

\section{Introduction}

Congenital heart disease (CHD) is reported with an incidence of 4 to 50 per 1,000 live births-depending primarily on the number of small ventricular septal defects included in the series [1] - and it is estimated that $85 \%$ of children with CHD will survive to adulthood due to improvements in medical management and surgical procedures [2]. Around $50 \%$ of these children will require follow-up in adult life [3]. Thus, precise follow-up imaging of these patients is important not only for monitoring the lesions and their functional status but also to provide accurate information on the anatomical relationship of the coronary, cardiac and extracardiac structures to plan surgical reintervention.

The traditional imaging technique for the pre- and postoperative evaluation of patients with CHD was catheter angiography, but this has been replaced by echocardiography for most conditions [4]. However, some anatomical structures, including the pulmonary vasculature, the aortic arch and the right ventricle, are difficult to evaluate with transthoracic echocardiography, while trans-oesophageal echocardiography is limited in cases of pulmonary enlargement [5]. Moreover, the diagnostic quality of echocardiography is highly dependent on the operator and the presence of an adequate acoustic window. Magnetic resonance imaging (MRI) has entered the diagnostic armamentarium in patients with CHD because of its non-invasiveness, the accurate morphological and functional information provided, and because neither an iodinated contrast agent nor ionising radiation is required. Therefore, MRI is an excellent diagnostic tool particularly for evaluation of young children with CHD who will require several follow-up examinations in their lifetime. However, many of the patients who undergo surgical correction of CHD have implanted pacemakers or 
cardioverter-defibrillators which preclude MRI, and the long examination time often requires a lengthy period of patient sedation. Moreover, the diagnostic capabilities of MRI are limited in the presence of surgical devices and for the evaluation of the airways and the lungs.

Computed tomography (CT) has undergone a rapid technical improvement in the last 10 years. Modern CT systems have high imaging speeds and high temporal and spatial resolution. Thus, CT combines the advantages of widespread availability and short acquisition times. When synchronised to the ECG signal, CT accurately delineates rapidly moving cardiac structures and allows assessment of the anatomical relationship of the coronary arteries to the adjacent structures and associated coronary artery anomalies [6]. The disadvantages of $\mathrm{CT}$ are the exposure of the patients to ionising radiation and the risks inherent in the application of iodinated contrast agents. Taking into account these drawbacks, patients should be carefully selected for CT imaging, and strategies for radiation exposure reduction need to be applied.

The purpose of this review is to discuss the relevant technical and imaging parameters for designing a CT protocol based on the suspected cardiac defect, to underscore strategies for reduction of radiation exposure and to describe the sequential segmental approach for the evaluation of CHD.

\section{CT imaging protocols}

All protocols and recommendations within this section are valid for a dual-source CT system but might be easily adapted to other CT systems.

The CT imaging protocol depends on the suspected cardiac defect, the type of previous surgical repair, and the patient's age and the level of cooperation (Table 1). The relevant $\mathrm{CT}$ parameters for CHD imaging in paediatric and adult patients are summarised in Table 2. In general, CT can be performed as a non-ECG-synchronised acquisition, or it can be synchronised to the ECG signal by retrospectively gated or prospectively triggered techniques. In most patients with CHD, non-ECG-synchronised CT is sufficient. However, in patients with a complex cardiac abnor mality or a small intracardiac abnormality or communication, synchronisation of the $\mathrm{CT}$ data acquisition to the ECG signal is recommended to reduce motion artefacts [7].

\section{Patient preparation}

Careful preparation is a prerequisite for CT in both paediatric and adult patients. We do not perform sedation in neonates. If sedation is necessary in children, we prefer a short-term sedation through the administration of midazolam hydrochloride $(0.1 \mathrm{mg} / \mathrm{kg}$ body weight $)$ via the intranasal route.

\section{Non-ECG-synchronised CT}

Non-ECG-synchronised CT provides a fast acquisition of the cardiac and extracardiac structures in patients with CHD but lacks the visualisation of small cardiac and coronary structures because of heart motion artefacts (Fig. 1). A pitch of around 1 to 1.5 usually represents a good balance of image quality, radiation dose and acquisition time provided. Thicker detector collimation is preferable for non-ECGsynchronised CT to reduce radiation dose and improve image quality impression at the expense of reduced detail visualisation of small structures. In addition, the required $\mathrm{CT}$ acquisition time is lower when using thicker collimation than for thin collimation imaging, and consequently respiratory motion artefacts might be reduced. However, if evaluation of small intracardiac structures is required, thin collimation should be used. In general, non-ECGsynchronised $\mathrm{CT}$ in CHD patients may be acquired during breath-hold. If the patient is not able to hold his or her breath, CT could be performed with a free-breathing technique but the patient should be advised to perform calm, shallow breathing and to avoid excessive respiratory excursions. However, respiratory artefacts are less remarkable with modern CT systems, and it has been reported that in $82 \%$ of patients with CHD the origins and proximal segments of the coronary arteries are evaluative in nonECG-synchronised CT acquisitions [8]. The radiation dose of this imaging technique is commonly less than $1 \mathrm{mSv}$ in young children [9].

\section{ECG-synchronised CT}

In retrospective ECG-gated $C T$ the data acquisition is performed with continuous imaging table motion and spiral acquisition with overlap of the radiation beam on each spiral synchronised to the ECG (Fig. 2). Hence, low pitch values are necessary for gapless data acquisition, and radiation dose is substantially higher than when using non-ECG-synchronised CT. However, this results in oversampling of information across different phases of the cardiac cycle and across several consecutive heart beats. Nevertheless, the synchronisation to the ECG signal provides artefact-free visualisation of cardiac and coronary structures even at elevated heart rates when using modern CT systems with high temporal resolution. A multisegment reconstruction algorithm using more than one heartbeat for image reconstruction might be applied to further improve temporal resolution. However, we usually prefer a monosegment algorithm because the multisegment algorithm 
Table 1 Recommended imaging range and ECG synchronisation depending on the congenital heart disease type

\begin{tabular}{|c|c|c|c|}
\hline $\begin{array}{l}\text { Congenital } \\
\text { heart } \\
\text { disease type }\end{array}$ & Imaging range & $\begin{array}{l}\text { ECG } \\
\text { synchronisation } \\
\text { required? }\end{array}$ & Comment \\
\hline $\begin{array}{l}\text { Aortic } \\
\text { coarctation }\end{array}$ & $\begin{array}{l}\text { Aortic arch to } \\
\text { diaphragm }\end{array}$ & No & $\begin{array}{l}\text { Thin collimation is recommended for } \\
\text { identification of collateral pathways }\end{array}$ \\
\hline $\begin{array}{l}\text { Anomalous } \\
\text { pulmonary } \\
\text { venous return }\end{array}$ & $\begin{array}{l}\text { Aortic arch to } \\
\text { diaphragm }\end{array}$ & No & $\begin{array}{l}\text { Imaging range should be extended to the } \\
\text { level of the kidneys in the infracardiac type }\end{array}$ \\
\hline $\begin{array}{l}\text { Patent ductus } \\
\text { arteriosus }\end{array}$ & $\begin{array}{l}\text { Aortic arch to } \\
\text { diaphragm }\end{array}$ & No & $\begin{array}{l}\text { Thin collimation is recommended for } \\
\text { identification of small ductus arteriosus }\end{array}$ \\
\hline $\begin{array}{l}\text { Persistent } \\
\text { superior left } \\
\text { vena cava }\end{array}$ & $\begin{array}{l}\text { Aortic arch to } \\
\text { diaphragm }\end{array}$ & No & - \\
\hline $\begin{array}{l}\text { Atrial septal } \\
\text { defect }\end{array}$ & $\begin{array}{l}\text { Below tracheal } \\
\text { bifurcation to } \\
\text { diaphragm }\end{array}$ & Yes & $\begin{array}{l}\text { ECG synchronisation may be beneficial for } \\
\text { small intracardiac shunts }\end{array}$ \\
\hline $\begin{array}{l}\text { Ventricular } \\
\text { septal defect }\end{array}$ & $\begin{array}{l}\text { Below tracheal } \\
\text { bifurcation to } \\
\text { diaphragm }\end{array}$ & Yes & $\begin{array}{l}\text { ECG synchronisation may be beneficial for } \\
\text { small intracardiac shunts }\end{array}$ \\
\hline $\begin{array}{l}\text { Tetralogy of } \\
\text { Fallot }\end{array}$ & $\begin{array}{l}\text { Above the } \\
\text { pulmonary } \\
\text { bifurcation to } \\
\text { diaphragm }\end{array}$ & No & - \\
\hline $\begin{array}{l}\text { Common } \\
\text { aortic- } \\
\text { pulmonary } \\
\text { trunk }\end{array}$ & $\begin{array}{l}\text { Above the } \\
\text { pulmonary } \\
\text { bifurcation to } \\
\text { diaphragm }\end{array}$ & No & - \\
\hline $\begin{array}{l}\text { Transposition } \\
\text { of the great } \\
\text { arteries }\end{array}$ & $\begin{array}{l}\text { Above the } \\
\text { pulmonary } \\
\text { bifurcation to } \\
\text { diaphragm }\end{array}$ & No & - \\
\hline $\begin{array}{l}\text { Univentricular } \\
\text { heart }\end{array}$ & $\begin{array}{l}\text { Below tracheal } \\
\text { bifurcation to } \\
\text { diaphragm }\end{array}$ & No & $\begin{array}{l}\text { No saline chasing bolus should be used to } \\
\text { avoid wash-out }\end{array}$ \\
\hline $\begin{array}{l}\text { Double outlet } \\
\text { ventricle }\end{array}$ & $\begin{array}{l}\text { Above pulmonary } \\
\text { bifurcation to } \\
\text { diaphragm }\end{array}$ & No & $\begin{array}{l}\text { No saline chasing bolus should be used to } \\
\text { avoid wash-out }\end{array}$ \\
\hline Isomerism & $\begin{array}{l}\text { Below tracheal } \\
\text { bifurcation to } \\
\text { diaphragm }\end{array}$ & Yes & $\begin{array}{l}\text { ECG synchronisation is recommended for } \\
\text { morphological identification of the atrial } \\
\text { appendages to determine sideness; the } \\
\text { imaging range must include the thorax and } \\
\text { spleen for identification of extracardiac } \\
\text { abnormalities }\end{array}$ \\
\hline $\begin{array}{l}\text { Coronary artery } \\
\text { anomaly }\end{array}$ & $\begin{array}{l}\text { Below tracheal } \\
\text { bifurcation to } \\
\text { diaphragm }\end{array}$ & Yes & $\begin{array}{l}\text { Thin collimation and ECG synchronisation } \\
\text { are mandatory }\end{array}$ \\
\hline
\end{tabular}

Table 2 Relevant CT parameters for imaging patients with congenital heart disease using a dual-source CT system

\begin{tabular}{lll}
\hline & Paediatric patients & Adult patients \\
\hline Slice collimation & Thin collimation: $2 \times 64 \times 0.6 \mathrm{~mm}$ \\
& Thick collimation: $2 \times 24 \times 1.2 \mathrm{~mm}$ \\
Tube voltage / tube current & $<20 \mathrm{~kg}: 80 \mathrm{kVp} / 50 \mathrm{mAs} \quad$ BMI $\leq 25 \mathrm{~kg} / \mathrm{m}^{2}: 100 \mathrm{kVp} / 150 \mathrm{mAs}$ \\
& $20-30 \mathrm{~kg}: 80 \mathrm{kVp} / 80 \mathrm{mAs} \quad \quad$ (ECG-synchronised: $100 \mathrm{kVp} / 220 \mathrm{mAs})$ \\
& $30-50 \mathrm{~kg}: 80 \mathrm{kVp} / 150 \mathrm{mAs} \quad$ BMI $>25 \mathrm{~kg} / \mathrm{m}^{2}: 120 \mathrm{kVp} / 180 \mathrm{mAs}$ \\
& $50-60 \mathrm{~kg}: 80 \mathrm{kVp} / 250 \mathrm{mAs} \quad \quad$ (ECG-synchronised: $120 \mathrm{kVp} / 330 \mathrm{mAs})$ \\
& $330 \mathrm{~ms}$ & \\
Gantry rotation time & 1 (non-ECG-synchronised CT) & \\
Pitch & $0.2-0.5$ (retrospective ECG-gated CT; pitch depending on the heart rate) \\
\hline
\end{tabular}




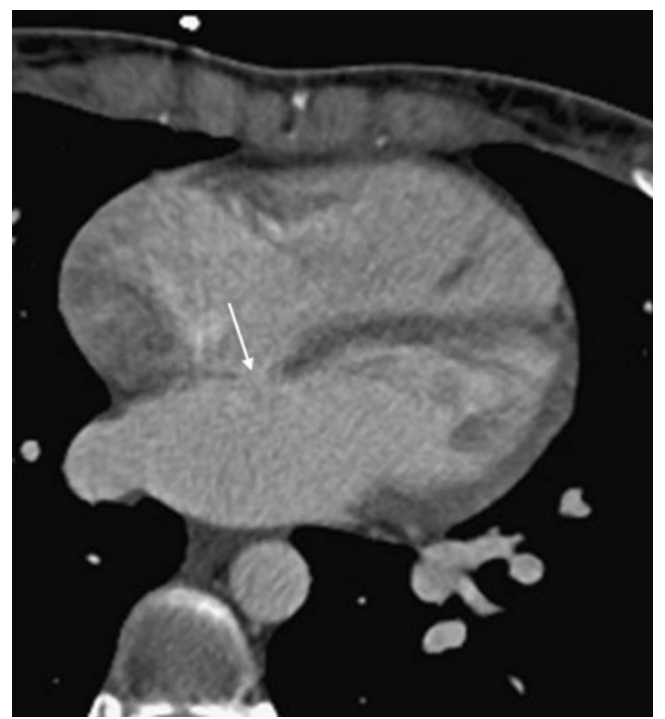

Fig. 1 Non-ECG-synchronised CT in a 24-year-old man demonstrating a large atrial septal defect (arrow)

prolongs the acquisition time and might introduce additional motion artefacts in the case of heart rate variability
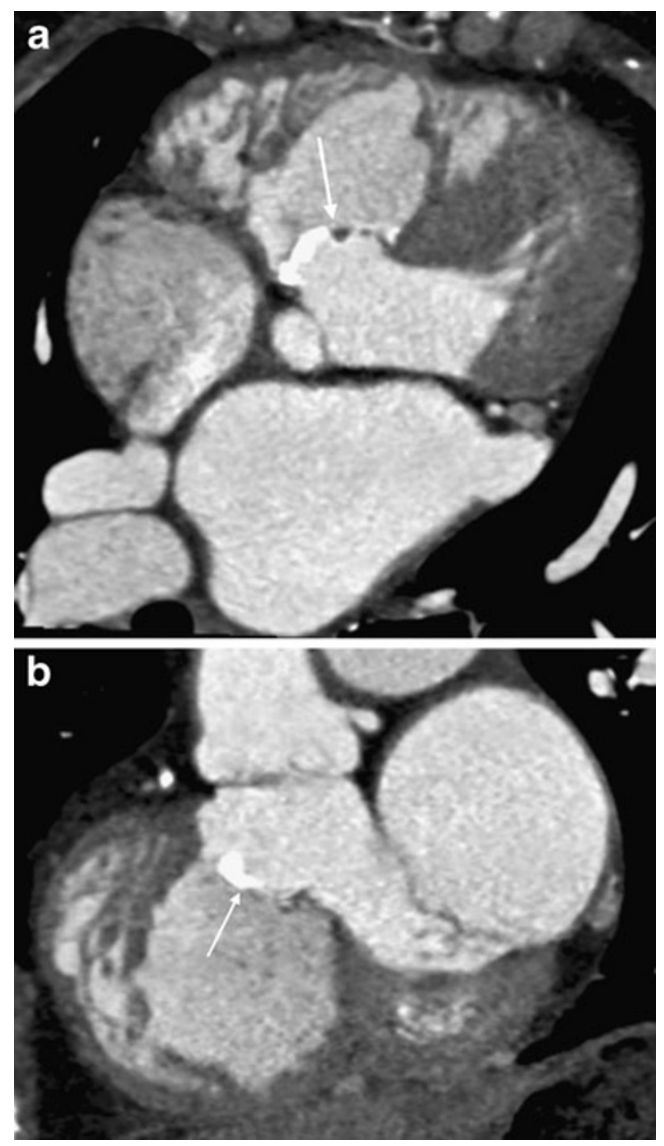

Fig. 2 Retrospective ECG-gated CT in a 14-year-old girl in a transverse (a) and short-axis reconstruction (b) performed for follow-up evaluation after surgical correction of a tetralogy of Fallot. The patch completely closes the ventricular septal defect (arrow). Note the overriding position of the aorta and the right ventricular hypertrophy
[10]. Using retrospective ECG-gated CT, the effective radiation dose is around $2-6 \mathrm{mSv}$ for patients with $\mathrm{CHD}$ [11]. Although ECG-synchronised CT allows for the assessment of left and right ventricular function, performing a non-ECG-synchronised CT for morphology and an additional echocardiography for functional information is usually the right way to make a diagnosis.

Conversely, in prospective ECG-triggered CT irradiation is exposed at user-selectable predefined heart phases only and the table is moved in a sequential manner with the table remaining stationary while data are acquired (Fig. 3). This technique has the potential of a smaller effective patient dose, which has been reported to be $1-3 \mathrm{mSv}$ in adults [12] and $0.2-0.7 \mathrm{mSv}$ in newborns and infants [11]. However, this technique is limited by the fact that information on ventricular function can not be assessed and image quality can be impaired in the case of arrhythmia. To date, prospective ECG-triggered $\mathrm{CT}$ has almost completely replaced retrospective ECG-gated acquisition in patients with CHD. For patients with heart rates $<75$ bpm, we predefine the data acquisition in the diastolic phase at $70 \%$ relative to the R-R interval and for patients with a heart rate of more than $75 \mathrm{bpm}$ in the systolic phase at $30 \%$. We still perform a retrospective ECG-gated CT-if ECGsynchronisation is required - in patients with arrhythmia and if multi-phase functional evaluation is required. Most recently an adaptation of the prospective ECG-triggered CT technique to a dual-step mode has been introduced to provide ventricular functional analysis at a low radiation dose [13].

Electrocardiography-synchronised CT is not commonly used in children because of the associated higher radiation dose. As the greater cardiac structures are affected by motion to a minor degree, ECG synchronisation is unlikely to contribute to improved image quality. On the other hand, ECG-synchronised CT is associated with a longer imaging time, and, therefore, motion artefacts due to respiration or body motion might be more crucial than the beneficial effects of cardiac motion suppression.

\section{High-pitch CT}

Recently, the dual-source technique has been refined to allow CT acquisitions at a high-pitch value of up to 3.4 [14]. In single-source CT, the applicable pitch is limited to 1.5 to ensure gapless volume coverage. In the high-pitch acquisition mode available at dual-source CT, the second detector system can be used to fill these gaps [14]. The high-pitch acquisition mode is fast enough to reduce the acquisition time for the entire chest to below $1 \mathrm{~s}$ [15] and therefore might allow artefact-free visualisation of the coronary arteries [15] and the aortic root [16] in routine non-ECG-synchronised chest CT studies or to compensate 

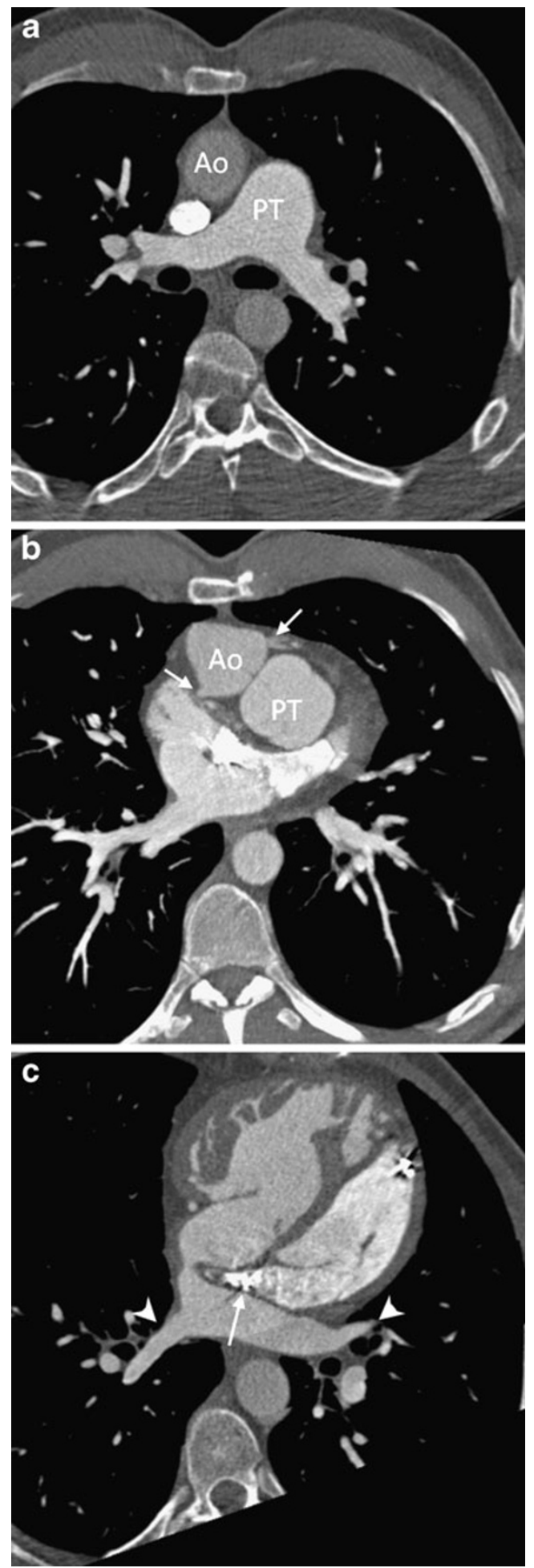

Fig. 3a-c Prospective ECG-gated CT in a 20-year-old man. Transverse sections at the level of the pulmonary trunk (a), the origin of the coronary arteries (arrows in b) and the ventricles (c) demonstrate levotransposition of the great arteries. The aorta $(A o)$ and the pulmonary trunk $(P T)$ are transposed with the aorta anterior and to the left of the pulmonary artery. Note the superior cava vein draining into the leftsided atrium (arrow) and the pulmonary veins draining into the rightsided atrium (arrowheads) for respiratory motion. In ECG-gated cardiac CT acquisitions, the high-pitch mode has been demonstrated to provide artefact-free visualisation of the coronary arteries at a radiation exposure below $1 \mathrm{mSv}[14,17]$. However, to the best of our knowledge this novel technique has not yet been investigated in CHD patients.

\section{Contrast agent application}

For contrast agent application, a right-arm injection is preferable to avoid high-contrast artefacts on the left brachiocephalic vein. For neonates and young children, non-ionic iodinated contrast agent is injected at a dose of $2 \mathrm{~mL} / \mathrm{kg}$ body weight to a maximum amount of $100 \mathrm{~mL}$. The injection rate is set at $1 \mathrm{~mL} / \mathrm{s}$ but can be increased to $2 \mathrm{~mL} / \mathrm{s}$ in patients with a large intracardiac communication. In adults, $1.5 \mathrm{~mL} / \mathrm{kg}$ body weight is injected at a rate of 3$4 \mathrm{~mL} / \mathrm{s}$. To reduce artefacts from undiluted contrast material and to reduce the total amount of contrast material, a saline bolus chasing technique should be applied. We prefer the bolus tracking technique to determine the imaging delay in ECG-synchronised CT. In the paediatric patient, a region of interest is placed in the left ventricle, and a threshold attenuation of $200 \mathrm{HU}$ is set. In adults, the region of interest is placed in the ascending aorta, and the attenuation threshold is set at $140 \mathrm{HU}$. However, when in doubt about the intracardiac connections and the timing of the contrast attenuation of the different cardiac chambers, monitoring the arrival of contrast material in the cardiac chambers and manually starting the CT acquisition is often the safer way for sufficient contrast attenuation of the cardiac cavities and adjacent arteries and veins. In non-ECG-synchronised CT in paediatric patients a fixed imaging delay is usually sufficient with the start delay set at $12 \mathrm{~s}$ for peripheral injection and $8 \mathrm{~s}$ for central venous injection.

\section{Image reconstruction}

Several image reconstruction techniques are available for the evaluation of the $\mathrm{CT}$ acquisition including multi-planar reformations (MPR), maximum intensity projections (MIP) and the volume rendering technique (VRT). In general, a combination of the $2 \mathrm{D}$ and $3 \mathrm{D}$ reconstruction techniques is used for the evaluation of CT in CHD patients. The 2D MPR is the most relevant reconstruction algorithm as it is capable of displaying the CT dataset in any plane and even in curved planes if desired. In general, the axial source images and coronal and sagittal MPR are reviewed to evaluate the cardiac and extracardiac structures. Oblique MPR are interactively arranged to review potential intracardiac connections or to evaluate the atrioventricular and ventriculo-arterial junctions. Three-dimensional MIP and VRT are used to image a portion or the complete dataset in 
one image, which is particularly helpful to get an overview of the cardiac and extracardiac structures and for preoperative planning and postoperative evaluation of the surgical procedures that have been performed.

\section{Strategies for reduction of radiation exposure}

Various techniques for reduction of the radiation dose are currently available for cardiac CT. Radiation dose reduction is of utmost importance particularly in paediatric CHD patients in whom several follow-up studies will be performed during their lifetime. According to the ALARA principle ("as low as reasonably achievable"), CT protocols and imaging parameters need to be adapted to result in the lowest possible radiation exposure without losing relevant information for diagnosis.

\section{Restriction of z-axis coverage}

The z-axis coverage of the CT acquisition needs to be limited to the relevant structures. One has to bear in mind that the CT radiation dose is directly proportional to the imaging range when all other acquisition parameters are similar, and that ECG-synchronised cardiac CT has the highest radiation dose per centimetre imaging range of all CT protocols. A recent study observed that, by minimisation of the imaging range in cardiac CT, $0.7 \mathrm{mSv}$ for each centimetre of the imaging range lost can be saved in adults when using a retrospective ECG-gated technique [18].

\section{Adaptation of imaging parameters to body size}

One of the easiest and most effective ways to limit radiation dose in CT for CHD imaging is to implement body-sizeadaptive CT protocols. Various parameters that reflect the body size might be used to adapt the imaging parameters. In our institution, we find it most practicable to adapt the imaging parameters to the body weight in paediatric patients, while in adults adaptation to the body mass index (BMI) is favourable. For that reason, CT in adults with a normal BMI of $\leq 25 \mathrm{~kg} / \mathrm{m}^{2}$ is performed with a tube voltage of $100 \mathrm{kVp}$ and a tube current time-product of $220 \mathrm{mAs} /$ rotation. In overweight patients with a BMI of $>25 \mathrm{~kg} / \mathrm{m}^{2}$ $\mathrm{CT}$ is performed at $120 \mathrm{kVp}$ and $330 \mathrm{mAs} /$ rotation. Using these adaptations in adults, a reduction in radiation dose of around $50 \%$ has been found for the low kilovoltage compared with the $120-\mathrm{kVp}$ standard protocol [19].

ECG-based tube current modulation

In order to reduce the radiation dose associated with retrospective ECG-gated CT, the tube current should be modulated according to the ECG (aka 'ECG pulsing'). With this technique, the tube current output outside a predefined phase in the cardiac cycle is reduced to $4-20 \%$ of the nominal tube current inside the pulsing window [19]. The image quality is impaired by noise outside the full tube current window, thereby rendering those phases of the cardiac cycle inadequate for detailed morphological evaluation but providing sufficient image quality for ventricular functional analysis. Optimal ECG pulsing windows for cardiac CT depend on the patient's heart rate and have been recommended as follows [20]: $60-70 \%$ of the R-R interval at heart rates of $\leq 60 \mathrm{bpm}, 60-80 \%$ at heart rates of 60 $70 \mathrm{bpm}, 55-80 \%$ at heart rates of $70-80 \mathrm{bpm}$, and $30-80 \%$ at heart rates of $>80 \mathrm{bpm}$. The ECG pulsing technique has been reported to reduce the radiation dose by up to $64 \%$ [21].

Considerations for $\mathrm{CT}$ imaging for CHD in paediatric patients

Radiation dose reduction is of highest importance in children because of greater radiosensitivity, a longer life expectancy and the possible requirement of several followup studies during their lifetime.

Computed tomography imaging for CHD in neonates and children should always be performed at $80 \mathrm{kVp}$. Cardiac CT with a tube voltage of $80 \mathrm{kVp}$ has been successfully performed in adults with a body weight below $60 \mathrm{~kg}$ [22]. In children, CT with $80 \mathrm{kVp}$ provides sufficient image quality when the tube current output is adapted to the patient's body weight (Table 2). Besides the advantage of saving radiation dose, the $80-\mathrm{kVp}$ setting allows the total amount of contrast agent to be reduced because iodine has a higher attenuation at lower energy. In addition, non-ECGsynchronised $\mathrm{CT}$ is the preferred acquisition technique in neonates and children because of higher heart rates in those patients and shorter acquisition times of non-ECGsynchronised $\mathrm{CT}$, which results in fewer respiratory artefacts. If an ECG-synchronisation technique is required, the CT protocol should be performed with a narrow ECG pulsing window width to limit the radiation exposure.

\section{Evaluation of CHD}

The interpretation of CT studies of CHD patients should be performed on dedicated workstations capable of multiplanar reformations (MPR), maximum-intensity projections (MIP) and volume rendering (VR) techniques. The primary review is performed on the axial source images. Most of the relevant information can be assessed on these images. Then, depending on the cardiac disorders and previous surgical procedures, the MPR planes need to be interac- 
tively adapted to the abnormal findings and the blood flow or they might be adjusted to display imaging planes analogous to those of echocardiography. MIP with variable slab thickness may be helpful in evaluating the great vessels. VR images are recommended in presurgical evaluation in order to demonstrate to the surgeon the relationship of anatomical structures.

The complex nature of most CHD and the fact that even simple CHD can cause alterations of several up- and downstream structures in the circulation often render correct diagnosis and reporting of abnormal findings in CHD challenging. Several approaches have been described to interpret the disorders. CHD might be described by embryological grading of the CHD according to the developmental period in which the disease occurred or described according to the clinical manifestation nomenclature differentiating CHD in cyanotic or acyanotic heart defects and the presence of left-right or right-left shunts. For the analysis of CHD, we prefer a sequential segmental approach [23-25]. Although introduced for echocardiography in the 1970s, this approach is ideally suited to CT diagnosis of CHD because it is based on the morphological appearance and helps to systematically review the CT study for pathological cardiac conditions. According to the sequential segmental approach, any heart can be considered in three segments: the atrial chambers, the ventricles and the great arteries. By examining the arrangement of the heart components and their connections, each case of CHD will be described in a sequential manner (Table 3).
In the first step, the arrangement of the atrial chambers (situs) has to be determined. The normal asymmetrical arrangement of the heart chambers is called the situs solitus. If the heart chambers are on the wrong side it is designated a situs inversus. In an isomeric arrangement two morphological right or left chambers are present. The sideness of the atria is defined by the morphology of the atrial appendages. The right atrial appendage is characterised by a triangular shape, a wide opening to the atrial chamber and large pectinate muscles. The left atrial appendage is more finger-like in shape with a narrow opening to the atrial chamber and has no or small pectinate muscles.

In the second step the morphology of the ventricles is analysed. The right ventricle shows a coarse trabecularisation. The papillary muscles root from the septum and a thin moderator band can be identified. In the left ventricle the trabecularisation shows a fine criss-cross pattern. Two papillary muscles are connected only to the parietal wall. The atrioventricular arrangement has to be assessed.

The third step of the sequential segmental analysis includes the determination of the great arteries and the ventriculoarterial junctions. A normal ventriculo-arterial junction is called a concordant junction with connection of the right ventricle to the pulmonary artery and the left ventricle to the aorta. A discordant junction describes a changed ventriculoarterial junction. The right ventricle is connected to the aorta and the left ventricle to the pulmonary artery. In the case of an ambiguous junction a one-side-changed ventriculo-arterial junction is found at isomerism.

Table 3 Examples of descriptions of common congenital heart disease in the segmental sequential nomenclature

\begin{tabular}{ll}
\hline CHD type & Sequential segmental analysis \\
\hline Ventricular septal defect & Normal atrial arrangement, concordant atrioventricular and ventriculo-arterial connections, levocardia \\
& + VSD \\
& Normal atrial arrangement, concordant atrioventricular and ventriculo-arterial connections, levocardia \\
& + Aortic coarctation \\
Aortic coarctation & Normal atrial arrangement, concordant atrioventricular and ventriculo-arterial connections, levocardia \\
& + VSD with pulmonary stenosis, overriding aorta, right ventricular hypertrophy \\
Tetralogy of Fallot & Normal atrial arrangement, concordant atrioventricular and discordant ventriculo-arterial connections, \\
& levocardia \\
Transposition of the great arteries with & + VSD \\
associated VSD & Normal atrial arrangement, discordant atrioventricular and discordant ventriculo-arterial connections, \\
& levocardia \\
Congenitally corrected transposition of & Normal atrial arrangement, concordant atrioventricular and double-outlet ventriculo-arterial connec- \\
the great arteries & tions from the right ventricle, levocardia \\
Double-outlet right ventricle & VSD \\
& Ambiguous atrial arrangement, ambiguous atrioventricular and ambiguous ventriculo-arterial \\
& connections, levocardia \\
& Bilateral bilobed lungs with hyparterial bronchus on both sides, polysplenia, interrupted inferior \\
& vena cava with azygos continuation
\end{tabular}

VSD Ventricular septal defect 
In the fourth step associated anomalies are assessed. In simple types of congenital heart disease the atrial and ventricular arrangement and the ventriculo-arterial junction are normal and the CHD type is then only determined by the extracardiac malformations including anomalies of pulmonary venous return, septal defects, coronary anomalies or anomalies of the aortic arch.

The fifth step describes the position of the heart within the chest and the orientation of the cardiac apex.

\section{Conclusion}

Although echocardiography and MRI are the primary imaging techniques for diagnosing the vast majority of congenital cardiac abnormalities, CT plays an increasingly complementary role by providing detailed morphological information particularly in the evaluation after surgery. However, in patients in whom no surgical or interventional procedures have been performed MRI is superior to CT in the comprehensive morphological and particularly functional evaluation of CHD. The CT imaging protocol depends on the suspected cardiac defect, the type of previous surgical repair, and the patient's age and level of cooperation. For most congenital cardiac disorders non-ECG-synchronised CT is adequate and the benefit of obtaining functional information with ECG-gated CT must outweigh the increased radiation exposure especially in young patients. Familiarity of the performing radiologist with dedicated $\mathrm{CT}$ protocols, the complex anatomy, morphology and terminology of CHD, as well as with the surgical procedures used to correct congenital abnormalities is a prerequisite for correct diagnosis.

\section{References}

1. Hoffman JI, Kaplan S (2002) The incidence of congenital heart disease. J Am Coll Cardiol 39:1890-1900

2. Moller JH, Taubert KA, Allen HD, Clark EB, Lauer RM (1994) Cardiovascular health and disease in children: current status. A Special Writing Group from the Task Force on Children and Youth, American Heart Association. Circulation 89:923-930

3. Wren C, O'Sullivan JJ (2001) Survival with congenital heart disease and need for follow up in adult life. Heart 85:438-443

4. Goo HW, Park IS, Ko JK, Kim YH, Seo DM et al (2003) CT of congenital heart disease: normal anatomy and typical pathologic conditions. Radiographics 23:S147-S165

5. Goo HW (2010) State-of-the-art CT imaging techniques for congenital heart disease. Korean J Radiol 11:4-18

6. Kacmaz F, Ozbulbul NI, Alyan O, Maden O, Demir AD et al (2008) Imaging of coronary artery anomalies: the role of multidetector computed tomography. Coron Artery Dis 19:203-209

7. Leschka S, Oechslin E, Husmann L, Desbiolles L, Marincek B et al (2007) Pre- and postoperative evaluation of congenital heart disease in children and adults with 64-section CT. Radiographics 27:829-846

8. Goo HW, Yang DH (2010) Coronary artery visibility in freebreathing young children with congenital heart disease on cardiac 64-slice CT: dual-source ECG-triggered sequential scan vs. singlesource non-ECG-synchronized spiral scan. Pediatr Radiol 40:1670-1680

9. Yang DH, Goo HW, Seo DM, Yun TJ, Park JJ et al (2008) Multislice CT angiography of interrupted aortic arch. Pediatr Radiol 38:89-100

10. Leschka S, Alkadhi H, Stolzmann P, Schmid FT, Leschka SC et al (2008) Mono- versus bisegment reconstruction algorithms for dual-source computed tomography coronary angiography. Invest Radiol 43:703-711

11. Goo HW, Seo DM, Yun TJ, Park JJ, Park IS et al (2009) Coronary artery anomalies and clinically important anatomy in patients with congenital heart disease: multislice CT findings. Pediatr Radiol 39:265-273

12. Stolzmann P, Leschka S, Scheffel H, Krauss T, Desbiolles L et al (2008) Dual-source CT in step-and-shoot mode: noninvasive coronary angiography with low radiation dose. Radiology 249:71-80

13. Feuchtner G, Gotti R, Plass A, Baumueller S, Stolzmann P et al (2010) Dual-step prospective ECG-triggered 128-slice dual-source CT for evaluation of coronary arteries and cardiac function without heart rate control: a technical note. Eur Radiol 20:2092-2099

14. Achenbach S, Marwan M, Schepis T, Pflederer T, Bruder H et al (2009) High-pitch spiral acquisition: a new scan mode for coronary CT angiography. J Cardiovasc Comput Tomogr 3:117-121

15. Goetti R, Baumuller S, Feuchtner G, Stolzmann P, Karlo C et al (2010) High-pitch dual-source CT angiography of the thoracic and abdominal aorta: is simultaneous coronary artery assessment possible? AJR Am J Roentgenol 194:938-944

16. Karlo C, Leschka S, Goetti RP, Feuchtner G, Desbiolles L et al (2011) High-pitch dual-source CT angiography of the aortic valveaortic root complex without ECG-synchronization. Eur Radiol 21:205-212

17. Leschka S, Stolzmann P, Desbiolles L, Baumueller S, Goetti R et al (2009) Diagnostic accuracy of high-pitch dual-source CT for the assessment of coronary stenoses: first experience. Eur Radiol 19:2896-2903

18. Leschka S, Kim CH, Baumueller S, Stolzmann P, Scheffel H et al (2010) Scan length adjustment of CT coronary angiography using the calcium scoring scan: effect on radiation dose. AJR Am J Roentgenol 194:W272-277

19. Stolzmann P, Scheffel H, Schertler T, Frauenfelder T, Leschka $S$ et al (2008) Radiation dose estimates in dual-source computed tomography coronary angiography. Eur Radiol 18:592-599

20. Leschka S, Scheffel H, Desbiolles L, Plass A, Gaemperli O et al (2007) Image quality and reconstruction intervals of dual-source CT coronary angiography: recommendations for ECG-pulsing windowing. Invest Radiol 42:543-549

21. Weustink AC, Mollet NR, Pugliese F, Meijboom WB, Nieman K et al (2008) Optimal electrocardiographic pulsing windows and heart rate: effect on image quality and radiation exposure at dualsource coronary CT angiography. Radiology 248:792-798

22. Abada HT, Larchez C, Daoud B, Sigal-Cinqualbre A, Paul JF (2006) MDCT of the coronary arteries: feasibility of low-dose CT with ECG-pulsed tube current modulation to reduce radiation dose. AJR Am J Roentgenol 186:S387-390

23. Van Praagh R (1972) The segmental approach to diagnosis in congenital heart disease. In: Bergsama D (ed) National Foundation-March of Dimes birth defects: original articles series, vol VIII, no 5. Williams \& Wilkins, Baltimore

24. Shinebourne EA, Macartney FJ, Anderson RH (1976) Sequential chamber localization - logical approach to diagnosis in congenital heart disease. Br Heart J 38:327-340

25. Anderson RH, Becker AE, Freedom RM, Macartney FJ, QueroJimenez $M$ et al (1984) Sequential segmental analysis of congenital heart disease. Pediatr Cardiol 5:281-287 